\title{
Comunicación
}

\section{Diámetro Biparietal en Fetos a Término en Varias Razas de Perros}

\author{
Biparietal Diameter in Foetuses at Final Stage of Gestation in \\ Dogs OF VARIOUS BREEDS
}

Jacqueline Cahua U.,2, Lizeth Quispe M. ${ }^{1}$

\section{Resumen}

El presente estudio tuvo por objetivo la determinación de la medición ecográfica cuantitativa del diámetro biparietal de fetos a término en perras. Se trabajó con 181 informes ecográficos de varias razas de perras en gestación cuyos fetos tenían menos de 210 latidos por minuto, y que fueron examinados en el consultorio de ecografía de la Clínica de Animales Menores de la Facultad de Medicina Veterinaria, Universidad Nacional Mayor de San Marcos, Lima, Perú, entre los años 2000-2014.

Palabras clave: DBP, diámetro biparietal, ultrasonografía, perra, raza

\section{Abstract}

The present study aimed to determine the ultrasonographic size of the biparietal diameter in foetuses at the final stage of gestation in dogs of various breeds. Sonographic reports $(n=181)$ of bitches in gestation where foetuses had less than 210 beats per minute were studied. The animals were attended at the Small Animal Clinic of the Faculty of Veterinary Medicine, San Marcos University, Lima, Peru from 2000 to 2014.

Key words: DBP, biparietal diameter, ultrasonography, bitch, breed

${ }^{1}$ Clínica de Animales Menores, Facultad de Medicina Veterinaria, Universidad Nacional Mayor de Marcos, Lima, Perú

${ }^{2}$ E-mail: jackiecahua@gmail.com

Recibido: 15 de abril de 2016

Aceptado para publicación: 13 de julio de 2016 


\section{INTRODUCCIÓN}

La gestación en la perra tiene un tiempo variable de 57 a 72 días después de la monta, 65 días desde la subida inicial de progesterona o pico de LH, 63 días a partir de la ovulación o 57 días desde el inicio del diestro (Galina y Valencia, 2009). La duración de la gestación se prolonga en camadas de 4 o menos crías (Eilts et al., 2005; Groppetti et al., 2015).

La distocia ocurre cuando el parto no sucede en la forma y tiempo normal, necesitando asistencia veterinaria. La inercia uterina primaria es la principal causa, pero también ocurre en partos de perras braquiocefálicas por desproporcionalidad fetopélvica, en perras con estrechez del canal pélvico y en gestaciones únicas por la desigualdad en proporción entre la pelvis de la madre y el tamaño del feto (Luvoni y Beccaglia de 2006; Socha et al., 2008). Se reporta que el diámetro biparietal (DBP) tiende a aumentar en gestaciones con feto único (Luvoni y Beccaglia, 2006). Asimismo, estas causas de distocia son menos frecuentes en razas gigantes, siendo más común en ellos la distocia por inercia uterina secundaria (Bergström et al., 2006).

La ecografía es una técnica no invasiva y segura, que se usa para el diagnóstico y evaluación de la preñez, para calcular la cantidad aproximada de fetos, su viabilidad y la estimación de la proximidad del parto a través de la determinación del DBP de los fetos (Socha et al., 2012). Asimismo, en el feto a término, una disminución en la frecuencia cardiaca de 180 a 220 lat/min indica un sufrimiento fetal leve y una frecuencia menor de $180 \mathrm{lat} / \mathrm{min}$ indica un severo sufrimiento fetal (Zone y Wanke, 2001).

Aunque e El DBP determinado mediante ecografía es importante para determinar la proximidad del parto, sus valores son dependientes de las razas. El objetivo del presente trabajo fue determinar el DBP por raza de fetos a término, con el fin de poder prede- cir una probable distocia, en especial por macrosomía fetal.

\section{Materiales y Métodos}

Se realizó un estudio retrospectivo tomando como referencia 181 archivos de informes ecográficos del servicio de ecografía de la Clínica de Animales Menores de la Facultad de Medicina Veterinaria, Universidad Nacional Mayor de San Marcos, correspondiente a los años 2000-2014. Los exámenes seleccionados incluyeron fetos a término de la gestación que presentaban latidos cardiacos menores de 210 lat $/ \mathrm{min}$, indicativo de un leve a marcado sufrimiento fetal (Zone y Wanke, 2001).

El estrés fetal es diagnosticado por una reducción de la frecuencia cardiaca debida a una hipoxia. La frecuencia cardiaca fetal normal es de 220 a 240 lat/min, mientras que frecuencias menores de 180 lat/min indican estrés fetal (Zone y Wanke, 2001).

Para la toma del diámetro biparietal se usó un ecográfo SonoAce Pico (Samsung Medison, Corea) con transductor microconvexo de 4-9 MHz. No se administraron drogas tranquilizantes, pero se usó una colchoneta cóncava que ayudó a inmovilizar a los animales. La evaluación de cada animal se hizo en cerca de 30 minutos y siempre fue ejecutada por dos de los médicos que trabajan en la sección de ecografía veterinaria.

\section{Resultados y Discusión}

El Cuadro 1 muestra los valores promedio del DBP fetal según la raza. El DBP del Yorkshire fue el de menor medida entre todas las razas $(22.8 \mathrm{~mm})$. No obstante, Son et al. (2001) reportaron que el Chihuahua es la raza que tiene el menor DBP $(24 \mathrm{~mm})$. Es posible, que los Yorkshire del país sean de menor tamaño que el promedio en otras latitudes. Por otro lado, la raza de mayor tama- 
Cuadro 1. Medidas promedio del diámetro parietal (DBP) obtenida mediante ecografía de fetos a término según raza de perros

\begin{tabular}{|c|c|c|c|c|c|c|}
\hline \multirow[b]{2}{*}{ Tamaño } & \multirow[b]{2}{*}{ Raza } & \multirow{2}{*}{$\begin{array}{l}\text { Examen } \\
\text { (n) }\end{array}$} & \multicolumn{3}{|c|}{ Diámetro biparietal (DBP) } & \multirow{2}{*}{$\begin{array}{l}\text { Promedio de } \\
\text { crías por } \\
\text { gestación }\end{array}$} \\
\hline & & & $\begin{array}{l}\text { Media } \\
(\mathrm{mm})\end{array}$ & d.e. & $\begin{array}{l}\text { Rango } \\
(\mathrm{mm})\end{array}$ & \\
\hline \multirow[t]{4}{*}{ Miniatura } & Yorkshire & 12 & 22.8 & 1.3 & $20-25$ & 3 \\
\hline & Chihuahua & 19 & 23.7 & 1.2 & $22-25.5$ & 3 \\
\hline & Pequinés & 13 & 24.1 & 1.3 & $22-26$ & 4 \\
\hline & Shitzu & 15 & 24.3 & 1.2 & $22-26$ & 4 \\
\hline \multirow[t]{6}{*}{ Pequeño } & Boston Terrier & 3 & 24.3 & 1.5 & $23-26$ & 5 \\
\hline & Fox Terrier & 4 & 25.2 & 0.9 & $24-26$ & 4 \\
\hline & Basset Hound & 5 & 25.6 & 1.1 & $24-27$ & 4 \\
\hline & Cocker Spaniel & 19 & 25.7 & 1.8 & $22-29$ & 4 \\
\hline & Schnauzer Toy & 7 & 25.9 & 1.2 & $24-28$ & 4 \\
\hline & Beagle & 15 & 26.7 & 1.5 & $24-28.5$ & 4 \\
\hline \multirow[t]{6}{*}{ Mediano } & Caniche Estándar & 9 & 25.1 & 1.5 & $23-27$ & 4 \\
\hline & Schnauzer Estándar & 10 & 26.6 & 0.9 & $25.6-28$ & 5 \\
\hline & Chow-Chow & 4 & 26.8 & 1.4 & $25-28$ & 5 \\
\hline & Bull Terrier & 12 & 27.4 & 1.3 & $26-30$ & 6 \\
\hline & Pitbull & 3 & 28.6 & 0.6 & $28-29.1$ & 5 \\
\hline & Bulldog Inglés & 13 & 29.2 & 0.9 & $28-30.8$ & 6 \\
\hline \multirow[t]{3}{*}{ Grande } & Rottweiller & 5 & 28.2 & 0.9 & $27-29.6$ & 5 \\
\hline & Samoyedo & 3 & 28.8 & 1.0 & $28-30$ & 4 \\
\hline & $\begin{array}{l}\text { Golden Retriever y } \\
\text { Labrador }\end{array}$ & 6 & 29.4 & 1.8 & $27-32$ & 5.5 \\
\hline Gigante & San Bernardo & 4 & 30.8 & 1.3 & $29-32$ & 3 \\
\hline Total & & 181 & & & & \\
\hline
\end{tabular}

ño de DBP fue el San Bernardo con 30.8 $\mathrm{mm}$.

La media del DBP del Golden Retriever fue de $29.4 \mathrm{~mm}$, muy parecida a la media encontrada por England et al. (1990). También se puede observar que el DBP del Bulldog Inglés, raza mediana pero de cabeza braquiocefálica, presenta un DBP mayor que el de las razas grandes, lo cual le predispone a la ocurrencia de distocias. En general, los resultados demuestran que el DBP de los fetos a término es mayor cuanto más grande es la raza del perro.

La desviación estándar en algunos casos fue menor de $1 \mathrm{~mm}$, demostrando una menor variabilidad y en todos los casos fue menor o igual a $1.8 \mathrm{~mm}$, lo cual demuestra que los DBP de los fetos a término no tienen mucha variación dentro de una misma raza. 


\section{Literatura Citada}

1. Bergström A, Nodtvedt A, Lagerstedt $A$, Egenvall $A$. 2006. Incidence and breed predilection for dystocia and risk factors for cesarean section in a Swedish population of insured dogs. Vet Surg 35: 786-791. doi:10.1111/j.1532-950x.2006. 00223.x

2. Eilts B, Davidson A, Hosgood G, Paccamonti D, Baker D. 2005. Factors affecting gestation duration in the bitch. Theriogenology 64: 242-251. doi: 10.1016/j.theriogenology.2004.11.007

3. England G, Allen W, Porter D. 1990. Studies on canine pregnancy using Bmode ultrasound: development of the conceptus and determination of gestational age. J Small Anim Pract 31: 324-329. doi: 10.1111/j.1748-5827.1990. tb00821.x

4. Galina C, Valencia J.2009. Reproducción de animales domésticos. $3^{\mathrm{a}}$ ed. México DF: Limusa. 582 p.

5. Groppetti D, Vegetti F, Bronzo V, Pecile A. 2015. Breed-specific fetal biometry and factors affecting the prediction of whelping date in the German shepherd dog. Anim Reprod Sci 152: 117-122. doi: 10.1016/j.anireprosci.2014.11.018

6. Luvoni GC, Beccaglia M. 2006. The prediction of parturition date in canine pregnancy. Reprod Dom Anim 41: 2732. doi: 10.1111/j.1439-0531.2006. 00641.x

7. Socha P, Rudowska M, Janowski T. 2012. Effectiveness of determining the parturition date in bitches using the ultrasonographic fetometry as compared to hormonal and cytological methods. Pol J Vet Sci 15: 447-453.

8. Socha P, Szczebiot A, Janowski T. 2008. New applications of ultrasonography in the diagnosis and control of pregnancy in bitches. Medycyna Wet 64: 1371-1374.

9. Son CH, Jeong K, Kim J, Park IC, Kim S, Lee CS. 2001. Establishment of the prediction table of parturition day with ultrasonography in small pet dogs. J Vet Med Sci 63: 715-721.

10. Zone M, Wanke M. 2001. Diagnosis of canine fetal health by ultrasonography. J Reprod Fertil 57: 215-219. 